\title{
Predictions from information-theoretical models of nonequilibrium radiation
}

\author{
J. Fort \\ Departament de Física, EPS, Universitat de Girona, 17071 Girona, Catalonia, Spain
}

(Received 21 August 1998)

\begin{abstract}
The radiation distribution function used by Domínguez and Jou [Phys. Rev. E 51, 158 (1995)] has been recently modified by Domínguez-Cascante and Faraudo [Phys. Rev. E 54, 6933 (1996)]. However, in these studies neither distribution was written in terms of directly measurable quantities. Here a solution to this problem is presented, and we also propose an experiment that may make it possible to determine the distribution function of nonequilibrium radiation experimentally. The results derived do not depend on a specific distribution function for the matter content of the system. [S1063-651X(99)10502-6]
\end{abstract}

PACS number(s): 05.30.-d, 44.40.+a, 05.70.Ln

Information statistical theory $[3,4]$ has been applied recently to generalize the Planck distribution to nonequilibrium systems $[1,2,5]$. This is a relevant problem because, in contrast with matter [6], a well-established nonequilibrium distribution for radiation that has been tested in the laboratory does not seem to be available at present. DomínguezCascante and Faraudo have presented a model in which the radiation distribution function follows from the maximization of the radiative entropy under the constraints of fixed energy density, fixed energy flux, and vanishing photon number flux [2]. This leads to the radiation distribution

$$
f_{r}=\frac{1}{\exp \left[\beta p_{r} c-\vec{\gamma} \cdot p_{r} c \vec{c}+\vec{\delta} \cdot \vec{c}\right]-1},
$$

where $p_{r}$ and $\vec{c}$ stand for the photon momentum and velocity, respectively, and $\beta, \vec{\gamma}$, and $\vec{\delta}$ are Lagrange multipliers. The motivation for requiring an additional constraint of vanishing photon number flux was that the authors of Ref. [2] noted that the distribution previously proposed by Domínguez and Jou, namely [1],

$$
\widetilde{f_{r}}=\frac{1}{\exp \left[\beta p_{r} c-\vec{\gamma} \cdot p_{r} c \vec{c}\right]-1},
$$

can also be obtained by performing a Lorentz boost to a frame in which the distribution is that of equilibrium [which corresponds to $\vec{\gamma}=0$ in Eq. (2)].

Neither of the proposals (1) and (2), as they stand, can give a complete description of the radiation emitted by a nonequilibrium system, for in Refs. [1,2] the Lagrange multipliers $\beta, \vec{\gamma}$, and $\vec{\delta}$ were not written in terms of directly measurable quantities. Here we will derive this dependence, and propose how it might be verified experimentally.

It has been recently argued by Nettleton [7] that statistical-mechanical models of radiation, as applied, e.g., by the authors of Refs. [1,2] in their proposals of the distributions (2) and (1), cannot be embodied into a thermodynamically consistent theory because of the nonlocality of the radiation field. We share his view, since the nonlocality of radiation transfer in vacuum may be seen as a consequence of the fact that photons do not interact with each other. This makes, in particular, the evolution of an isolated system towards equilibrium impossible. Therefore, as pointed out by Landau and Lifshitz [8], in a nonequilibrium theory, it is necessary to consider a system composed of matter in addition to radiation $[9,10]$. Moreover, if a photon has momentum $\vec{p}_{r}$, we cannot localize it in space. Let us, therefore, consider a macroscopically small system, centered at point $\vec{R}$, within which the matter has an approximately uniform temperature. The neighboring regions feed a radiant flux into the system. If the density of matter is high enough, photons will be emitted and absorbed in a highly localized region. Under such circumstances, and assuming for the moment that the matter part of the system is a classical ideal gas, the entropy density of the system can be written as $[8,11]$

$$
\begin{aligned}
\tilde{s}= & \tilde{s}_{m}+\tilde{s}_{r}=-k \int \frac{d^{3} p_{m}}{h^{3}} f_{m} \ln f_{m} \\
& +2 k \int \frac{d^{3} p_{r}}{h^{3}}\left[\left(1+f_{r}\right) \ln \left(1+f_{r}\right)-f_{r} \ln f_{r}\right],
\end{aligned}
$$

where the subindexes $m$ and $r$ stand for matter and radiation, $\vec{p}$ and $f=f(\vec{R}, \vec{p})$ are the corresponding momenta and singleparticle distributions [11], respectively, $k$ is the Boltzmann constant, and $h$ is the Planck constant. The entropy (3) has been recently used in the study of nonequilibrium radiation [10,12-14]. However, Eq. (3) corresponds to a very special system. In contrast, the Planck distribution describes the equilibrium radiation emitted by any blackbody, independently of its composition and state. Thus any theory based on Eq. (3) is much less general, simply because interactions among particles cause (in nondilute systems) the matter entropy not to depend only on the single-particle distribution function: in general, one must work with the phase-space distribution $\rho_{m}[15]$. We are, therefore, lead to generalize Eq. (3) by (see also, Refs. $[16,17])$

$$
\begin{aligned}
\tilde{s}= & -k \int \frac{d \Gamma_{m}}{h^{3 N} V} \rho_{m} \ln \rho_{m} \\
& +2 k \int \frac{d^{3} p_{r}}{h^{3}}\left[\left(1+f_{r}\right) \ln \left(1+f_{r}\right)-f_{r} \ln f_{r}\right],
\end{aligned}
$$

where the first integration is over phase space [18], $V$ is the volume of the system considered above, and the problem posed is to determine the radiation distribution under the following constraints: 


$$
\begin{gathered}
\tilde{u}=\tilde{u}_{m}+\tilde{u}_{r}=\int \frac{d \Gamma_{m}}{h^{3 N} V} H_{m}\left(\Gamma_{m}\right) \rho_{m}+2 \int \frac{d^{3} p_{r}}{h^{3}} p_{r} c f_{r}, \\
\left\langle A_{l}\right\rangle=\int \frac{d \Gamma_{m}}{h^{3 N} V} A_{l}\left(\Gamma_{m}\right) \rho_{m}, \\
\vec{F}=2 \int \frac{d^{3} p_{r}}{h^{3}} p_{r} c \vec{c} f_{r} \\
\overrightarrow{0}=2 \int \frac{d^{3} p_{r} \vec{c} f_{r} .}{h^{3}}
\end{gathered}
$$

Here $\tilde{u}$ is the energy density, $\vec{F}$ is the radiative energy flux, and $H_{m}\left(\Gamma_{m}\right)$ is the microscopic operator corresponding to the matter Hamiltonian, e.g., for a single fluid with a pairwise potential energy $V_{i j}(\vec{r})$, one would have $H_{m}\left(\Gamma_{m}\right)$ $=\sum_{i=1}^{N}\left[p_{m i}^{2} / 2 m+\frac{1}{2} \sum_{j \neq i} V_{i j}\left(\vec{r}_{m j}-\vec{r}_{m i}\right)\right]$, with $m$ the molecular mass, $\vec{r}_{m i}$ and $\vec{p}_{m i}$ the position and momentum of the matter particle $i$, and $N$ the number of particles $[16,19,20]$. $A_{l}\left(\Gamma_{m}\right)$ are any additional operators corresponding, e.g., to the matter number density, to the conductive flux, etc. The constraint (8) corresponds to the requirement of vanishing photon number flux, in agreement with the point raised in Ref. [2]. Maximization of the entropy density (4) under the constraints (5) $-(8)$ yields

$$
\rho_{m}=\exp \left[-1-\beta H_{m}\left(\Gamma_{m}\right)-\sum_{l} \lambda_{l} A_{l}\left(\Gamma_{m}\right)\right],
$$

and Eq. (1) for $f_{r}$. The set $\left\{\lambda_{l}\right\}$ are Lagrange multipliers. The simplest case for the matter part is that of a classical monatomic ideal gas at rest and with negligible heat conduction, for which Eq. (9) becomes $\rho_{m}=Z \exp$ $\left[-\beta \sum_{i=1}^{N} p_{m i}^{2} / 2 m\right]$, where $Z$ normalizes $\rho_{m}$ to unity: this case corresponds to the single-particle Maxwellian distribution, namely, $f_{m}=C \exp \left[-\beta p_{m}^{2} / 2 m\right]$, where $C$ is such that $\int d^{3} p_{m} f_{m}=n$, with $n$ the molecular number density. We stress that, without need to consider such a specific matter content of the system, the radiation distribution is given by Eq. (1). However, we would like to remark that Eq. (1) will not hold unless the matter is sufficiently dense: if the matter were extremely dilute, photons reaching any small region of the system would have been emitted by matter at a different, completely independent temperature. When maximizing the total entropy of such a system, volume integrals would appear in the exponents in Eq. (9) $[19,21,22]$, so that the distribution modulus of radiation would be different from that of matter (i.e., $\beta_{r} \neq \beta_{m}$ ). Such a case would certainly complicate any thermodynamically consistent approach, as well as the derivation of any specific prediction. This is in agreement with the remarks by Essex [23] and Nettleton [7] on the thermodynamical implications of the nonlocality of radiative transfer in vacuum.

In order to obtain testable predictions, it is necessary to relate the Lagrange multipliers $\beta, \vec{\gamma}$, and $\vec{\delta}$ to measurable quantities. The identification of the Lagrange multiplier $\beta$ is based on the thermodynamical definition of temperature $T$, which identifies $T^{-1}$ with the partial derivative of the entropy with respect to the energy of the system [4]. It is pre- cisely this definition that allowed Boltzmann to derive the Stefan radiation law from thermodynamics and classical electrodynamics [24]. The derivation is done in the usual way [12] and yields

$$
\beta=\frac{1}{k T} .
$$

Let us, for the sake of mathematical simplicity, restrict our attention to near-equilibrium states. We assume that near-equilibrium states correspond to small values of the radiation multipliers $\vec{\gamma}$ and $\vec{\delta}$, an ansatz to be checked $a$ posteriori. By performing a MacLaurin expansion of the distribution (1) up to first order in $\vec{\gamma}$ and $\vec{\delta}$, we obtain

$$
\begin{aligned}
f_{r}= & \frac{1}{\exp \left[\beta p_{r} c\right]-1} \\
& \times\left(1+\frac{\exp \left[\beta p_{r} c\right]}{\exp \left[\beta p_{r} c\right]-1} p_{r} c \vec{c} \cdot \vec{\gamma}-\frac{\exp \left[\beta p_{r} c\right]}{\exp \left[\beta p_{r} c\right]-1} \vec{c} \cdot \vec{\delta}\right) .
\end{aligned}
$$

Use of this equation into the constraint (8) yields

$$
\vec{\delta}=\frac{18 \xi(3)}{\pi^{2} \beta} \vec{\gamma}
$$

where $\xi(z)$ is the Riemann zeta function, and the integrals have been performed by making use of the formulas 3.423,2 and 9.542,1 in Ref. [25]. Making use of Eqs. (10) and (12) into $\mathrm{Eq}$. (11), we may write the near-equilibrium radiation distribution as

$$
\begin{aligned}
f_{r}= & \frac{1}{\exp \left[\frac{p_{r} c}{k T}\right]-1} \\
& \times\left\{1+\left(p_{r} c-\frac{18 \xi(3) k T}{\pi^{2}}\right) \frac{\exp \left[\frac{p_{r} c}{k T}\right]}{\exp \left[\frac{p_{r} c}{k T}\right]-1}(\vec{c} \cdot \vec{\gamma})\right\} .
\end{aligned}
$$

Use of Eq. (13) into Eq. (7) and integration yields

$$
\vec{F}=4\left(\frac{1}{3}-\frac{135}{\pi^{6}}[\xi(3)]^{2}\right) a c^{2} k T^{5} \vec{\gamma},
$$

where $a=8 \pi^{5} k^{4} / 15 c^{3} h^{3}$ is the blackbody constant. In order to relate $\vec{\gamma}$ to the temperature gradient, we first calculate the pressure tensor of radiation, i.e., [26], $\stackrel{\vec{P}}{P}=2 \int_{R^{3}}\left(d^{3} p_{r} /\right.$ $\left.h^{3}\right)\left(p_{r} / c\right) \vec{c} \vec{c} f_{r}$, making use of Eq. (13). It is easily seen that the integrals of the terms in $(\vec{c} \cdot \vec{\gamma})$ vanish, and

$$
\vec{P}=\frac{a T^{4}}{3}\left(\begin{array}{lll}
1 & 0 & 0 \\
0 & 1 & 0 \\
0 & 0 & 1
\end{array}\right),
$$

where use has been made of formula 3.411.1 in Ref. [25]. We now assume, in order to obtain relatively simple expres- 
sions, that the system is in a steady state. As it is well known $[27,9]$, from the gray radiative transfer equation, it follows that $c\left(\partial P x i / \partial x+\partial P_{y i} / \partial y+\partial P z i / \partial z\right)=-\sigma F_{i}$, with $\sigma$ the absorption coefficient and $i=x, y, z$. Therefore, use of Eqs. (15) and (14) allows us to relate $\vec{\gamma}$ to measurable quantities,

$$
\vec{\gamma}=-\frac{1}{1-\frac{405}{\pi^{6}}[\xi(3)]^{2}} \frac{1}{\sigma c k T^{2}} \vec{\nabla} T .
$$

The intensity (per unit solid angle) of radiation is related to the photon distribution function through $I_{\nu}=\left(2 h \nu^{3} / c^{2}\right) f_{r}$ [28]. Thus, making use of Eqs. (13) and (16) and expressing the intensity per unit wavelength $\left(\lambda=c / \nu=c h / p_{r} c\right)$,

$$
\begin{aligned}
I_{\lambda}= & \frac{2 h c^{2}}{\lambda^{5}} \frac{1}{\exp \left[\frac{h c}{k T \lambda}\right]-1}\left(1-\frac{\frac{h c}{\lambda}-\frac{18 \xi(3) k T}{\pi^{2}}}{1-\frac{405}{\pi^{6}}[\xi(3)]^{2}}\right. \\
& \left.\times \frac{1}{\sigma k T^{2}} \frac{\exp \left[\frac{h c}{k T \lambda}\right]}{\exp \left[\frac{h c}{k T \lambda}\right]-1}(\hat{\Omega} \cdot \vec{\nabla} T)\right),
\end{aligned}
$$

where $\hat{\Omega}=\vec{c} / c$ is a unit vector.

We will now make a proposal in order to test this result experimentally. We also find it interesting to compare it with the one that follows from the maximization of the entropy density (4) under the constraints (5)-(7), without including the constraint (8). This yields the distribution (2) (which was considered in Ref. [1]) instead of Eq. (1). By repeating the same calculations as above, one finally finds

$$
\begin{aligned}
\tilde{I}_{\lambda}= & \frac{2 h c^{2}}{\lambda^{5}} \frac{1}{\exp \left[\frac{h c}{k T \lambda}\right]-1} \\
& \times\left(1-\frac{h c}{\lambda} \frac{1}{\sigma k T^{2}} \frac{\exp \left[\frac{h c}{k T \lambda}\right]}{\exp \left[\frac{h c}{k T \lambda}\right]-1}(\hat{\Omega} \cdot \vec{\nabla} T)\right),
\end{aligned}
$$

which is the intensity corresponding to near-equilibrium diffusion theory. In contrast with the intensity (17) (which has been derived here for the first time), Eq. (18) has been previously derived, both phenomenologically $[29,26]$ and from information theory [12].

In order to illustrate the predictions of the result (17), and to compare it with the previously derived intensity (18), we consider a system with a nonuniform temperature distribution. Such a system is depicted in Fig. 1. In the special case of a uniform temperature, the radiation would be Planckian, in agreement with either Eq. (17) or Eq. (18) for the special case of equilibrium $(\vec{\nabla} T=\overrightarrow{0})$,

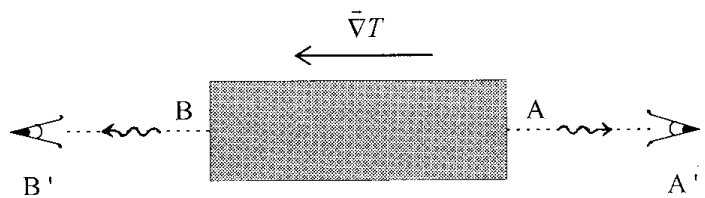

FIG. 1. Experiment proposed in order to determine the distribution function of nonequilibrium radiation. The system considered has a nonuniform temperature distribution. In Figs. 2 and 3 we present the predictions for the spectra of thermal radiation emitted in the directions $A$ and $B$ (such spectra can be measured by means of spectrophotometers located, e.g., at points of observation $A^{\prime}$ and $\left.B^{\prime}\right)$.

$$
I_{\lambda \mathrm{eq}}=\frac{2 h c^{2}}{\lambda^{5}} \frac{1}{\exp \left[\frac{h c}{k T \lambda}\right]-1} .
$$

We will consider this equilibrium result for comparison purposes only. The problem is then to determine (i) whether the predictions of Eq. (17) differ enough from the equilibrium result (19) so that such differences may be measured experimentally, and (ii) whether Eq. (17) changes the predictions of Eq. (18) in such a way that the experiment proposed in Fig. 1 allows us to determine which one of them, if any, corresponds to the physical reality. In order to answer both questions, we consider the radiation leaving the system in Fig. 1 in the direction of the temperature gradient [i.e., direction $B$ in Fig. 1, which corresponds to $(\hat{\Omega} \cdot \vec{\nabla} T)=|\vec{\nabla} T|]$ and the opposite direction [i.e., direction $A$ in Fig. 1, which corresponds to $(\hat{\Omega} \cdot \vec{\nabla} T)=-|\vec{\nabla} T|]$. In Fig. 2 we plot the spectra for the case $T_{A}=2000 \mathrm{~K}, T_{B}=2001 \mathrm{~K}$, a uniform temperature gradient of $|\vec{\nabla} T|=5 \mathrm{~K} / \mathrm{m}$, and $\sigma=0.1 \mathrm{~m}^{-1}$ [12], as predicted by Eq. (17) and also by Eq. (18). The dashed

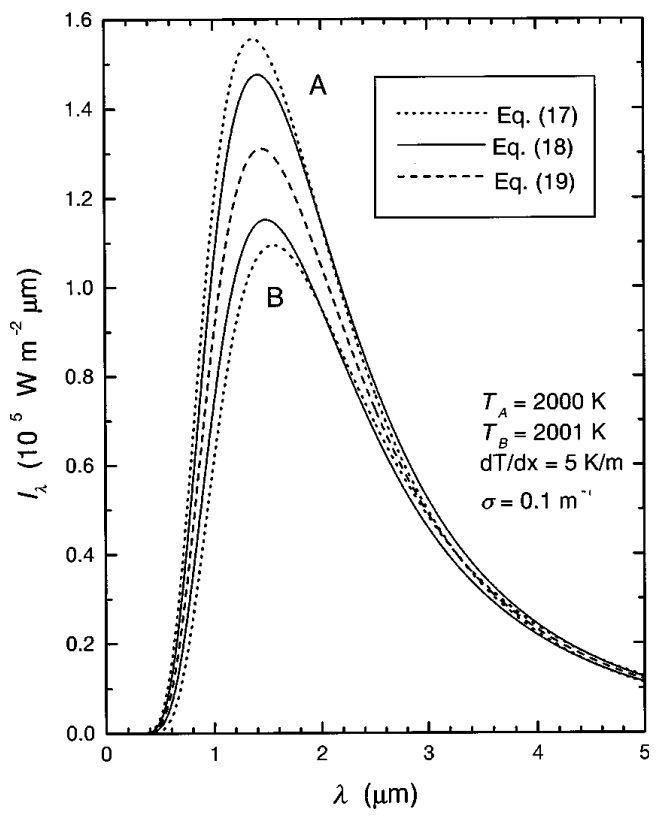

FIG. 2. Comparison between the spectra predicted by the distribution function derived in the present paper (dotted curves) and by near-equilibrium diffusion (full curves), for the experiment proposed in Fig. 1. In this figure, the dashed line is a Planckian spectrum (either at temperature $T_{A}$ or $T_{B}$ ). 


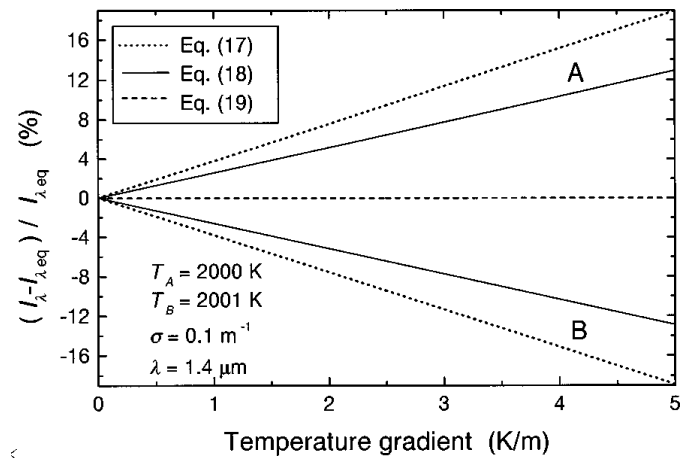

FIG. 3. Corrections to the Planckian intensity as a function of the temperature gradient, for the experiment proposed in Fig. 1. These corrections have been computed according to the distribution function derived in the present paper [the dotted curves are plots of $\left.\left.\left(I_{\lambda}-I_{\lambda \text { eq }}\right) / I_{\lambda \text { eq }}\right) \times 100\right]$ and to near-equilibrium diffusion theory [the full curves are plots of $\left.\left(\widetilde{I}_{\lambda}-I_{\lambda \text { eq }}\right) / I_{\lambda \text { eq }} \times 100\right]$.

line in Fig. 2 corresponds to the equilibrium, or Planckian, approximation (19). From Fig. 2, it is seen that the corrections of near-equilibrium diffusion theory [Eq. (18)] are as large as $13 \%$. On the other hand, the vanishing-photon-flux model derived in the present paper [Eq. (17)] yields an additional correction of about $6 \%$. The difference between both models is thus important enough so that such an experiment should make it possible to determine which one, if any, of both intensities corresponds to nonequilibrium radiation.

Because Fig. 2 depicts a very specific case, in Fig. 3 we present the correction with respect to the equilibrium approximation in terms of the temperature gradient. In thermal equilibrium $(|\vec{\nabla} T|=0)$, the intensity is Planckian [see Eq. (19)] and there is no correction. The higher the temperature gradient, the larger the corrections are.

Let us finally mention that, in spite of what is claimed in Ref. [2], it is not clear that one can conclude that Eq. (2) corresponds to equilibrium simply because this distribution acquires a Planckian form in a specific frame. According to Eq. (18), such radiation is emitted by matter with $\vec{\nabla} T \neq 0$, i.e., it corresponds to a nonequilibrium system. Moreover, if observers in Fig. 1 are in motion (relative to the emitting system), they can certainly determine whether this motion is consistent or not with a Planckian emission in the rest frame of the emitting system. Such points clearly show that theoretical attempts to solve the problem of nonequilibrium radiation via information theory (e.g., Refs. [1, 2, 12], and the present paper) would really benefit from an experimental approach to this fundamental question.

The author is very pleased to thank D. Jou, J. E. Llebot, and P. Roura for their comments. Computing equipment used in this paper has been partially funded by the DGICYT of the Ministry of Education and Culture under Grant No. PB 96-0451.
[1] R. Domínguez and D. Jou, Phys. Rev. E 51, 158 (1995).

[2] R. Domínguez-Cascante and J. Faraudo, Phys. Rev. E 54, 6933 (1996).

[3] E. T. Jaynes, Phys. Rev. 106, 620 (1957); 108, 171 (1957).

[4] D. Jou, J. Casas-Vázquez, and G. Lebon, Extended Irreversible Thermodynamics (Springer, Berlin, 1996).

[5] J. Camacho, Phys. Rev. E 51, 220 (1995).

[6] S. Chapman and T. G. Cowling, The Mathematical Theory of Non-Uniform Gases (Cambridge University Press, Cambridge, 1970).

[7] R. E. Nettleton, Phys. Rev. E 53, 1241 (1996).

[8] L. D. Landau and E. M. Lifshitz, Statistical Physics. Part 1 (Pergamon, Oxford, 1980).

[9] J. Fort and J. E. Llebot, J. Phys. A 29, 3427 (1996).

[10] J. Fort and J. E. Llebot, J. Math. Phys. 39, 345 (1998).

[11] In order to clarify the notation, we mention that we follow the approach in [8]: for example, $f_{m}$ stands for the number of matter particles in $d^{3} p_{m}$ and $d V$, divided by $d^{3} p_{m} d V / h^{3}$. In this way $\ln f_{m}$ and $\ln f_{r}$ are dimensionless and, e.g., the Maxwell distribution for a gas at rest reads $f_{m}$ $=h^{3} n /(2 \pi m k T)^{3 / 2} \exp \left[-p_{m}^{2} / 2 m k T\right]$ : see [8], Sec. 38 .

[12] J. Fort, Physica A 243, 275 (1997).

[13] J. Fort, D. Jou, and J. E. Llebot, Physica A 248, 97 (1998).

[14] J. Fort, J. A. González, and J. E. Llebot, Phys. Lett. A 236, 193 (1997).

[15] R. E. Nettleton and S. L. Sobolev, J. Non-Equilib. Thermodyn. 20, 205 (1995).
[16] D. Jou, C. Pérez-García and J. Casas-Vázquez, J. Phys. A 17, 2799 (1984).

[17] R. E. Nettleton, Phys. Rev. A 42, 4622 (1990).

[18] $\rho_{m}$ is defined as the number of microstates in $d \Gamma_{m}$ that correspond to a given macrostate, divided by the total number of microstates corresponding to the same macrostate, and multiplied by $h^{3 N}$. In this way, $\ln \rho_{m}$ is dimensionless. Similarly, $\ln f_{m}$ in Eq. (3) and $\ln f_{r}$ are also dimensionless [11].

[19] R. E. Nettleton, S. Afr. J. Phys. 14, 27 (1991).

[20] P. Résibois and M. De Leener, Classical Kinetic Theory of Fluids (Wiley, New York, 1977).

[21] R. E. Nettleton, J. Chem. Phys. 106, 10311 (1997).

[22] R. E. Nettleton, Period. Polytech., Chem. Eng. 41, 175 (1997).

[23] G. C. Essex, Advances in Thermodynamics (Taylor \& Francis, New York, 1990), Vol. 3, p. 435.

[24] A. Sommerfeld, Thermodynamics and Statistical Mechanics (Academic Press, San Diego, 1995).

[25] I. S. Gradshteyn and I. M. Ryzhik, Tables of Integrals, Series and Products (Academic Press, New York, 1984).

[26] G. C. Pomraning, The Equations of Radiation Hydrodynamics (Pergamon, Oxford, 1973).

[27] G. C. Pomraning, J. Quant. Spectrosc. Radiat. Transf. 27, 517 (1982).

[28] J. Oxenius, Kinetic Theory of Particles and Photons (Springer, Berlin, 1986).

[29] D. Mihalas and B. W. Mihalas, Foundations of Radiation Hydrodynamics (Oxford University Press, Oxford, 1984). 